\title{
Brote de COVID-19 en una comunidad indígena urbana en Lima Norte, Perú
}

\author{
COVID-19 outbreak in an urban indigenous community in Northern Lima, Peru \\ Luis Fernando Llanos Zavalaga ${ }^{1,2, a, b, c}$, Claudio Willbert Ramírez Atencio 1,3a,d,e, \\ Yanet Rocío Chura-Centeno ${ }^{1, a, b}$, Irving Brian Pareja-Palomino ${ }^{1, a, b}$, Henry Francisco Aguado Taquire ${ }^{1,4, a, f}$
}

\section{RESUMEN}

Las poblaciones en condiciones de pobreza, con movilidad social restringida y limitado acceso a servicios de salud, como la comunidad Shipibo-Konibo de Cantagallo (Rímac) son grupos vulnerables a COVID-19. Ante el reporte de 3 casos fallecidos por COVID-19, se desarrolló un trabajo articulado con participación intersectorial (Salud, Interior, Cultura), Municipalidad del Rímac, comunidad y ONG, entre otros; no permitiendo el ingreso ni salida de una persona sin autorización, cercándola y aislándola. Entre el primer y tercer tamizaje, la proporción de pacientes con IgM positivo disminuyó y la proporción de casos IgG positivo aumentó, sugiriendo disminución de la transmisión activa o presencia de casos leves o asintomáticos. La vulnerabilidad de comunidades originarias y nuestros resultados evidencian la necesidad de contar con planes articulados sostenibles, que trascienda del enfoque biomédico abordando los determinantes sociales, con activa participación intersectorial, intergubernamental y de sociedad civil, para hacer a COVID-19 en las comunidades indígenas.

PALABRAS CLAVE: Infecciones por coronavirus, poblaciones originarias, Perú. (Fuente: DeCS BIREME).

\section{SUMMARY}

Populations that live in poverty with restricted social mobility and limited access to health care services, such as the Shipibo-Konibo community of Cantagallo (Rimac), are at risk to get COVID-19. After the report of three fatalities due to COVID-19 in that community a multidisciplinary team involving the ministries of health, interior affairs, culture and the major of the Rimac district, the community of that district and NGOs did not allow the movement of persons in and out the community. The proportion of members of the community with positive IgM serological tests come down and those with positive IgG levels increased, suggesting reduced transmission or presence of mildasymptomatic cases. The vulnerability of indigenous communities as reported here is a clear evidence of the need to have sustainable and articulated plans to protect these communities from devastating diseases such as COVI-19.

KEYWORDS: Coronavirus Infections, indigenous population, Peru. (Source: MeSH NLM).

\footnotetext{
Dirección de Redes Integradas de Salud Lima Norte, MINSA

Universidad Peruana Cayetano Heredia.

Universidad Jorge Basadre Grohmann de Tacna

Universidad Peruana Los Andes

Médico Cirujano

Especialista en Administración en Salud

Maestría en Políticas, Planificación y Financiamiento en Salud.

Doctor en Medicina mención con Ciencias de la Salud Pública

Magister en Salud Pública

Egresado de la maestría en Medicina con mención en epidemiología.
} 


\section{INTRODUCCIÓN}

La COVID-19 es producida por el nuevo coronavirus SARS-CoV-2, y es una amenaza pública global meses después de varios casos a fines de 2019 en Wuhan, China ${ }^{(1)}$, siendo declarada por la Organización Mundial de la Salud (OMS) como una pandemia ${ }^{(2)}$. Al 14 de octubre 2021, el reporte de la COVID-19 de Universidad Johns Hopkins muestra que Perú reporta una tasa de mortalidad por 100000 habitantes de 614,41 y tasa de letalidad de $9,1 \%$, siendo las más altas a nivel mundial ${ }^{(3)}$.

La principal estrategia para reducir el impacto de la COVID-19 es la supresión ${ }^{(4)}$, que consiste en una combinación de políticas sanitarias para revertir el aumento de casos y mantener el número total de infecciones en niveles bajos para evitar el colapso de servicios de salud hasta tener una vacuna disponible. Esta restricción de libertades individuales se aplica en muchos países fomentando el distanciamiento social y decretando diferentes grados de confinamiento domiciliario. A ello se añaden la búsqueda activa de casos y contactos, así como el apoyo socio-económico de las personas vulnerables para sobrellevar el periodo de aislamiento y la pérdida de empleo relacionadas.

Prem et al. ${ }^{(5)}$, señalan que "las medidas extremas de distanciamiento físico, que incluyen cierres de escuelas, cierres de lugares de trabajo y evitar reuniones públicas a la vez, pueden impulsar la transmisión en hogares, aumentando allí los casos". Distinguir entre contactos repetidos y nuevos es importante en la propagación de enfermedades en modelos de redes de contactos.

El 11 de marzo de 2020, se declaró emergencia sanitaria a nivel nacional dictando medidas de prevención y control para la COVID-19 (6), con confinamiento obligatorio hasta junio de dicho año. Pese a las medidas tomadas por el Gobierno Peruano, la pandemia de COVID-19 se ha convertido en un problema de salud pública ocasionando, hasta el 27 de agosto de 2020, 613378 casos confirmados y 28124 fallecidos (letalidad 4,59\%), a nivel nacional ${ }^{(7)}$.

Se reconoce que un grupo vulnerable es la población en condiciones de pobreza o pobreza extrema, que viven en condiciones de hacinamiento y pobre acceso a agua potable, con movilidad social restringida y limitado acceso a servicios de salud (por barreras geográficas y culturales), aumentando su vulnerabilidad una vez que aparecen los casos iniciales. Ello ocurrió en la comunidad Shipibo-Konibo asentada en Cantagallo, distrito del Rímac desde el 2000, cuando 14 familias Shipibo-Konibo procedentes de la región Ucayali llegaron a Lima. Esta se caracteriza por su compleja dinámica cultural, economía y relaciones con la sociedad limeña. Fue urbanizada con casas de triplay y calles sin asfaltar, cuenta con un colegio bilingüe con 230 alumnos para el aprendizaje del español y el shipibo ${ }^{(8)}$.

En el 2012, la Municipalidad Metropolitana de Lima presentó el proyecto Rio Verde que consistía en reubicar a los habitantes que viven en Cantagallo y poder construir el Parque del Rímac, como parte de la recuperación del río Rímac; sin embargo, en 2015, se decidió cancelar este proyecto, la comunidad indígena se quedó sin poder reubicarse. Además, en noviembre de 2016 dicha comunidad fue consumida por un incendio que afectó 480 casas y dejó a 500 familias damnificadas.

\section{PRESENTACIÓN DEL CASO}

Ante el reporte de tres casos fallecidos por la COVID-19 en la comunidad Shipibo-Konibo de Cangallo; esta quedó convertida en un gueto sanitario, donde no se permitía el ingreso ni la salida de una persona sin autorización, cercando y aislando a esta población del resto de la ciudad ${ }^{(9)}$. Para ello, se desarrolló un trabajo articulado con participación de los sectores Salud, Interior, Cultura, la Municipalidad del Rímac, las organizaciones no gubernamentales (ONG), entre otros. Personal de la Policía Nacional resguardaba el área para reducir el riesgo de contaminación de la población residente. Equipos del Ministerio de Cultura se encargaron de implementar carpas y proporcionar alimentos a la comunidad. El equipo de Dirección de Monitoreo y Gestión Sanitaria de la Dirección de Redes Integradas de Salud (DIRIS) Lima Norte del Ministerio de Salud se encargó de implementar diversas acciones como parte de las estrategias desarrolladas para mejorar la vigilancia, prevención y control de la COVID-19 en esta población; del mismo modo, se contó con el apoyo del equipo de la Dirección de Salud Ambiental e Inocuidad Alimentaria (DSAIA), a través del equipo humanitario para el recojo de cadáveres. Además, hubo comunicación con representantes de la Pontifica Universidad Católica del Perú, de la Universidad Peruana Cayetano Heredia, y las ONG quienes se comprometieron a apoyar brindando equipos de protección personal y medicamentos para la atención de la población por problemas de salud. 
Tabla 1. Intervenciones sanitarias en la Comunidad Shipibo-Konibo realizadas por la DIRIS Lima Norte, mayo - agosto 2020.

\begin{tabular}{|c|c|c|c|c|c|c|c|c|c|c|}
\hline \multirow{3}{*}{ Fecha } & \multicolumn{6}{|c|}{ Positivo } & \multirow{2}{*}{\multicolumn{2}{|c|}{ Negativo }} & \multirow{2}{*}{\multicolumn{2}{|c|}{ Indeterminado }} \\
\hline & \multicolumn{2}{|c|}{ IgG } & \multicolumn{2}{|c|}{ IgM } & \multicolumn{2}{|c|}{ Mixto } & & & & \\
\hline & 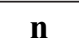 & $\%$ & n & $\%$ & $\mathrm{n}$ & $\%$ & n & $\%$ & $\mathbf{n}$ & $\%$ \\
\hline $\begin{array}{l}02 \text { mayo } \\
(\mathrm{n}=119)\end{array}$ & 5 & $4,20 \%$ & 0 & $0,00 \%$ & 35 & $29,41 \%$ & 79 & $66,39 \%$ & 0 & $0,00 \%$ \\
\hline $\begin{array}{l}12 \text { mayo } \\
(\mathrm{n}=475)\end{array}$ & 0 & $0,00 \%$ & 19 & $4,00 \%$ & 329 & $69,26 \%$ & 126 & $26,53 \%$ & 1 & $0,21 \%$ \\
\hline $\begin{array}{l}10 \text { agosto } \\
(\mathrm{n}=485)\end{array}$ & 237 & $48,87 \%$ & 3 & $0,62 \%$ & 90 & $18,56 \%$ & 155 & $31,96 \%$ & 0 & $0,00 \%$ \\
\hline
\end{tabular}

Según datos del último censo autogenerado en la Comunidad Shipibo-Konibo de Cantagallo, se reportan 273 viviendas, 295 familias y una población entre 1475 y 1550 habitantes. Al tomar conocimiento de los fallecidos y existencia de ciudadanos de esta comunidad que presentaban síntomas respiratorios sugestivos de infección respiratoria por SARS-CoV-2, se realizaron una serie de intervenciones sanitarias a cargo de los equipos de respuesta rápida de la DIRIS Lima Norte, quienes realizaron el tamizaje de pruebas rápidas y brindaron tratamiento médico a los casos confirmados de COVID-19. Para ello, también se contó con la colaboración de una estudiante de enfermería perteneciente a la misma comunidad que ayudó a reportar tempranamente la presencia de personas con síntomas sugestivos de la COVID-19.

En la primera intervención, realizada el 2 de mayo del 2020, se tamizaron personas sintomáticas o con antecedente de contacto con un paciente positivo. Se realizaron pruebas rápidas con anticuerpos a 119 miembros de la comunidad; de ellos, 40 resultaron positivos (tasa de positividad: $33,61 \%$ ), siendo $5 \mathrm{IgG}(+)$ y 35 positivos mixto. En vista al elevado porcentaje de positividad y a las condiciones de hacinamiento y confinamiento en que viven los miembros de esta comunidad, la DIRIS Lima Norte realizó el 12 de mayo del 2020 una segunda intervención sanitaria con donde se realizaron 475 pruebas rápidas, de las cuales resultaron $348(73,26 \%)$ positivas $(19 \operatorname{IgM}(+)$ y 329 positivos mixto).

Frente al elevado porcentaje de positividad y las condiciones de hacinamiento la DIRIS Lima Norte instaló un módulo de atención para seguimiento clínico a pacientes con COVID-19, quienes de forma diaria evaluaron signos y síntomas de la enfermedad en todos los pacientes identificados como positivos; y entregaron medicamentos según el protocolo establecido por el Ministerio de Salud. La atención se brindó hasta la primera semana de junio del 2020.

En seguimiento de las acciones implementadas, se realizó una tercera intervención sanitaria el 17 de agosto del 2020, a un tercer grupo de población no testeada previamente. En ella, se realizaron 485 pruebas rápidas, resultando 330 positivos ( 3 positivos a IgM, 237 positivos a IgG y 90 positivos mixto), tal como se muestra en la tabla 1.

Dentro de las acciones implementadas por la DIRIS Lima Norte, se realizó la difusión de medidas de bioseguridad para prevenir el contagio por la COVID-19, el tamizaje de pruebas rápidas para lograr un diagnóstico oportuno y el tratamiento temprano de los casos que resultaron positivos.

Se debe reconocer que luego de la implementación de las intervenciones articuladas, sólo se reportaron dos nuevos casos de fallecimiento por COVID-19 reportados al equipo humanitario de recojo de cadáveres de la DIRIS Lima Norte.

\section{DISCUSIÓN}

La migrante comunidad Shipibo-Konibo asentada en Cantagallo, distrito del Rímac, sigue conservando su cultura (su identidad, organización, lengua, arte, conocimientos ancestrales, entre otros). Estas migraciones son un acto social inducido por necesidades diversas y producen una forma de integración a una estructura organizacional urbana $(10,11)$.

Se reconoce que las muertes iniciales reportadas en la comunidad Shipibo-Konibo de Cantagallo 
permitió visibilizar a esta comunidad amazónica, muchas veces excluida e invisibilizada por la sociedad peruana. Un efecto de esta realidad fue el impulso del trabajo articulado intersectorial e intergubernamental sostenido, acompañado de la implementación de medidas estrictas de supresión, expresadas en lo que algunos llamaron un gueto sanitario ${ }^{(8)}$. Ello dio como resultado que no se reporten muertes como consecuencia de la COVID-19 en esta comunidad, hasta la fecha de presentación del artículo.

Existen diversas posibilidades que podrían explicar este logro. Primero, fue resultado del tamizaje temprano, o de la concientización de la población sobre la importancia de las medidas preventivas, o del tratamiento con ivermectina o hidroxicloroquina, tal como lo establecen las normas del MINSA para el tratamiento en el primer nivel de atención ${ }^{(12)}$.

Segundo, saliendo de una aproximación biomédica, este logro fue el resultado de una intervención articulada y sostenida entre los diversos sectores, los diversos niveles de gobierno y la sociedad civil. Tercero, es consecuencia de que la población Shipibo-Konibo es una población más joven que la población limeña y cuyos reportes de diabetes, hipertensión, obesidad y dislipidemia muestran prevalencias menores que el promedio limeño, tal como lo confirma el estudio de percepciones sobre COVID-19 realizado en julio del 2020 en dicha comunidad, reportando que en una muestra, la edad promedio fue 38,36 años, $63,2 \%$ de los entrevistados refirieron tener grado de instrucción secundaria y $52,6 \%$ refirieron que su ocupación primaria es artesanía ${ }^{(13)}$. Estas características de la población harían que tengan menor riesgo de desarrollar cuadros clínicos moderados a severos que la población general; pese a ser vulnerables por las condiciones socio-económicas, de exclusión social y limitada accesibilidad a servicios de salud de los miembros de la comunidad son un determinante que podría haber influido en la elevada tasa de ataque inicialmente reportada. Este estudio transversal impide identificar una causalidad.

Del análisis de la secuencia de tamizajes realizados, se observa que entre el primer tamizaje y el tercer tamizaje la proporción de pacientes con IgM positivo ha ido disminuyendo, mientras que la proporción de pacientes con IgG positivo esté aumentando nos sugeriría que afortunadamente, la enfermedad en esta población está pasando. Como resultado de las intervenciones implementadas, el estudio del CENSI ${ }^{(13)}$ reporta que $43,4 \%$ de la población fueron medicados por el equipo de salud, 59\% usaban mascarilla al momento de la entrevista; mientras que $83 \%$ referían usarla al salir de casa y $68 \%$ la usan cuando tienen un familiar infectado. El 64\% refirió que la mascarilla es comprada o donada.

En los modelamientos de transmisión de la enfermedad se debe considerar la diversidad de contactos en términos de intensidad y duración; una mayor intensidad y duración del contacto aumentaría el riesgo de severidad del cuadro clínico de la población contagiada. A esta condición de exposición se añadiría los diversos factores de riesgo que puede presentar la persona expuesta. Para ello, se deben implementar estrategias para asegurar un cumplimiento adecuado del aislamiento, incluyendo las medidas de control de infecciones en el hogar ${ }^{(5)}$.

Finalmente, se reconoce la biodiversidad cultural del país y la existencia de 51 pueblos indígenas amazónicos ${ }^{(14)}$. Las organizaciones de pueblos indígenas $\mathrm{u}$ originarios evidencian su temor ante la pandemia con permanentes pronunciamientos e impidiendo el ingreso de otra población a estas comunidades. La vulnerabilidad de las comunidades originarias y los resultados de esta intervención nos permiten establecer la necesidad de contar con un plan articulado y sostenido, intersectorial, intergubernamental y con activa participación de la sociedad civil, para la vigilancia, prevención y control de la COVID-19 en comunidades indígenas. Dicho plan debe incluir estrategias participativas de que trasciendan del enfoque biomédico y aborden los determinantes sociales. En este contexto, el Gobierno Peruano emitió el Decreto Legislativo $\mathrm{N}^{\circ} 1489$, que establece Acciones para la Protección de los Pueblos Indígenas u Originarios en el marco de la Emergencia Sanitaria declarada por el COVID-19 ${ }^{(15)}$.

\section{Declaración de financiamiento y de conflicto de intereses:}

El estudio fue financiado por los autores. Declaran no tener conflictos de interés.

\section{Contribución de autoría:}

LFLZ: Participó en el diseño de estudio, recolección de datos, redacción de artículo, responsabilidad de la integridad científica del contenido del artículo, 
aprobación final. CWRA: revisión crítica del artículo; YRCC: redacción del artículo; IBPP: redacción del artículo, revisión crítica del artículo; HFAT: revisión crítica del artículo, recolección de datos, aprobación final.

\section{Correspondencia:}

Luis Fernando Llanos Zavalaga

Dirección: Calle Las Lilas 435 - Urb. San Eugenio. Lince

Teléfono: (51) 999660680

Correo electrónico: luis.1lanos@upch.pe

\section{REFERENCIAS BIBLIOGRÁFICAS}

1. Wang L, Wang Y, Ye D, Liu Q. Review of the 2019 Novel Coronavirus (COVID-19) based on current evidence. Int J Antimicrob Agents. 2020; 55(6):105948. doi: 10.1016/j.jjantimicag.2020. 105948

2. Organización Panamericana de la Salud. La OMS caracteriza a COVID-19 como una pandemia [Internet]. (Citado el 13 octubre de 2021). Disponible en: https://www.paho.org/es/noticias/11-3-2020oms-caracteriza-covid-19-como-pandemia

3. Johns Hopkins University. Coronavirus Resource Center. [Internet] (Citado el 13 octubre de 2021). Disponible en: https://coronavirus.jhu.edu/data/ mortality

4. Ferguson NM, Laydon D, Nedjati-Gilani G, et al. Impact of Non-pharmaceutical Interventions (NPIs) to ReduceCOVID-19mortality andhealthcaredemand. Londres: Imperial College COVID-19 Response Team; 2020. (Citado el 24 de setiembre del 2020) Disponible en: https://www.imperial. ac.uk/media/imperial-college/medicine/sph/ide/ gida-fellowships/Imperial-College-COVID19-NPImodelling-16-03-2020.pdf

5. Prem K, Liu Y, Russell TW, Kucharski AJ, Eggo RM, Davies N. The effect of control strategies to reduce social mixing on outcomes of the COVID-19 epidemic in Wuhan, China: a modelling study. Lancet. 2020; 5(5):E261-E270.

6. Presidencia de la Republica. Decreto Supremo $\mathrm{N}^{\circ}$ 008-2020-SA Perú. Lima: Diario Oficial El Peruano; 11 de marzo del 2020 (Citado el 24 de setiembre del 2020) Disponible en: https://busquedas.elperuano. pe/normaslegales/decreto-supremo-que-declara-enemergencia-sanitaria-a-nivel-decreto-supremo-n008-2020-sa-1863981-2/
7. Centro Nacional de Epidemiologia, Prevención y Control Enfermedades. Sala Situacional COVID-19 Perú. Lima: Ministerio de Salud; 2020. (Citado el 24 de setiembre del 2020) Disponible en: https:// covid19.minsa.gob.pe/sala_situacional.asp

8. Defensoría del Pueblo. Cronología: El caso de la comunidad indígena urbana Shipibo-Konibo de Cantagallo.: Defensoría del Pueblo; noviembre 2016. (Citado el 24 de setiembre del 2020) Disponible en: https://www.defensoria.gob.pe/blog/cronologiacaso-comunidad-indigena-urbana-shipibo-konibode-cantagallo/

9. RPP. "Nadie entra, nadie sale": el drama en la comunidad shipiba de Cantagallo por la COVID-19. Lima:RPP; 2020. (Citado el 24 de setiembre del 2020) Disponible en: https://rpp.pe/lima/actualidad/nadieentra-nadie-sale-el-drama-en-la-comunidad-shipibade-cantagallo-por-la-covid-19-fotos-noticia-1265782

10. Maidana CA. Migración Indígena, Procesos de Territorialización y Análisis de Redes Sociales. REMHU. 2013; 21(41):277-93.

11. Cárdenas EP. Migración interna e indígena en México: enfoques y perspectivas. Intersticios Sociales. 2014; 7:1-28.

12. Ministerio de Salud. Resolución Ministerial $\mathrm{N}^{\circ}$ 270-2020-MINSA. Modificatoria de RM No ${ }^{\circ} 193$ Documento Técnico: Prevención Diagnóstico y Tratamiento de Personas afectadas por COVID-19. Lima: Diario Oficial El Peruano; 08 de mayo 2020. (Citado el 24 de setiembre del 2020) Disponible en: https://cdn.www.gob.pe/uploads/document/ file/694719/RM_270-2020-MINSA.PDF

13. Instituto Nacional de Salud. Informe final: Percepción sobre COVID-19 y medidas de protección en pobladores Shipibo-Konibo residentes en Cantagallo. Lima: Instituto Nacional de Salud; 2020.

14. Ministerio de Cultura. Base de datos de Pueblos Indígenas u Originarios. Lima: Ministerio de Salud; 2020 (Citado el 24 de setiembre del 2020) Disponible en: Instituto Nacional de Salud. http://bdpi.cultura. gob.pe/pueblos-indigenas

15. Poder Ejecutivo. Decreto Legislativo $\mathrm{N}^{\circ} 1489$, Decreto Legislativo que establece Acciones para la Protección de los Pueblos Indígenas u Originarios en el marco de la Emergencia Sanitaria declarada por el Covid-19. Lima: Diario Oficial El Peruano; 09 de mayo 2020. (Citado el 27 agosto del 2020). Disponible en: https://busquedas.elperuano.pe/download/url/ decreto-legislativo-que-establece-acciones-para-laproteccio-decreto-legislativo-n-1489-1866212-1

Recibido: 27/09/2020

Aceptado: 21/10/2021 